\title{
PSEUDO-UMBILICAL SURFACES WITH CONSTANT GAUSS CURVATURE
}

\author{
by BANG-YEN CHEN $\dagger$ \\ (Received 21st October 1971)
}

\section{Introduction}

Let $M$ be a surface immersed in an $m$-dimensional space form $R^{m}(c)$ of curvature $c=1,0$ or -1 . Let $\boldsymbol{h}$ be the second fundamental form of this immersion; it is a certain symmetric bilinear mapping $T_{x} \times T_{x} \rightarrow T_{x}^{1}$ for $x \in M$, where $T_{x}$ is the tangent space and $T_{x}^{1}$ the normal space of $M$ at $x$. Let $\boldsymbol{H}$ be the mean curvature vector of $M$ in $R^{m}(c)$ and $\langle$,$\rangle the scalar product on R^{m}(c)$. If there exists a function $\lambda$ on $M$ such that $\langle\boldsymbol{h}(\boldsymbol{X}, \boldsymbol{Y}), \boldsymbol{H}\rangle=\lambda\langle\boldsymbol{X}, \boldsymbol{Y}\rangle$ for all tangent vectors $X, Y$, then $M$ is called a pseudo-umbilical surface of $R^{m}(c)$. Let $D$ denote the covariant differentiation of $R^{m}(c)$ and $\eta$ be a normal vector field. If we denote by $D^{*} \eta$ the normal component of $D \eta$, then $D^{*}$ defines a connection in the normal bundle. A normal vector field $\boldsymbol{\eta}$ is said to be parallel in the normal bundle if $D^{*} \eta=0$. The length of mean curvature vector is called the mean curvature.

Let $e$ be a unit normal vector at $x \in M$ in $R^{m}(c)$. Then the second fundamental form $\boldsymbol{h}(\boldsymbol{e})$ at $\boldsymbol{e}$ is defined by $\langle\boldsymbol{h}, \boldsymbol{e}\rangle$; it is a certain symmetric bilinear mapping $T_{x} \times T_{x} \rightarrow \mathbb{R}$. Let $h_{i j}^{r} ; i, j=1,2 ; r=3, \ldots, m$ be the coefficients of the second fundamental form $\boldsymbol{h}$ (for the details, see $\S 2$ ). Then the Gauss curvature $K$ and the normal curvature $K_{N}$ are given respectively by

$$
\begin{gathered}
K=\sum_{r=3}^{m}\left(h_{11}^{r} h_{22}^{r}-h_{12}^{r} h_{12}^{r}\right), \\
K_{N}=\sum_{r, s=3}^{m}\left[\sum_{k=1}^{2}\left(h_{1 k}^{r} h_{2 k}^{s}-h_{2 k}^{r} h_{1 k}^{s}\right)\right]^{2} .
\end{gathered}
$$

The mean curvature vector $\boldsymbol{H}$, the Gauss curvature $K$ and the normal curvature $K_{N}$ play the most important rôles, in differential geometry, for surfaces in space forms.

Theorem 1. Let $M$ be a pseudo-umbilical surface with constant Gauss curvature in a space form $R^{m}(c)$ of curvature $c$. If the mean curvature is constant and the normal curvature $K_{N}$ vanishes, then $M$ is either flat or totally umbilical in $R^{m}(c)$. In particular, if $c \geqq 0$, then $M$ is either totally umbilical or contained in a Clifford torus.

$\dagger$ This work was supported in part by the National Science Foundation under Grant GU-2648. 
A minimal surface of a sphere $S^{m-1} \subset E^{m}$ is a pseudo-umbilical surface with constant mean curvature in $E^{m}$, and the normal curvature of a surface in $S^{3} \subset E^{4}$ is zero. Therefore by Theorem 1 , we have the following strong result.

Corollary (5). Let $M$ be a minimal surface of a 3-sphere $S^{3}$ with constant Gauss curvature. Then $M$ is either totally geodesic or contained in a Clifford torus in $S^{3}$.

Remark 1. If the assumption that $K_{N}=0$ is omitted, then Theorem 1 is no longer true. The Veronese surface in a euclidean space and the hyperbolic Veronese surface in a hyperbolic space are examples of pseudo-umbilical surfaces in space forms with constant Gauss curvature, constant mean curvature but with normal curvature $K_{N} \neq 0$ (see, for instance (2), (4)).

Let $e$ be a unit normal vector field of $M$ in $R^{m}(c)$. If $e$ is parallel in the normal bundle and the determinant of $h(e)$ is nowhere zero, then $e$ is called a non-degenerate normal vector field. For a compact surface with Gauss curvature $K \leqq 0$, we have the following flatness theorem.

Theorem 2. Let $M$ be a compact surface with Gauss curvature $K \leqq 0$ in a space form $R^{m}(c)$. If there exists a non-degenerate normal vector field perpendicular to the mean curvature vector field, then $M$ is flat and the normal curvature $K_{N}$ vanishes.

Remark 2. For minimal surfaces with Gauss curvature $\leqq 0$, see (1). For surfaces with mean curvature vector parallel in the normal bundle, see (3).

\section{Preliminaries}

Let $M$ be a surface immersed in an $m$-dimensional space form $R^{m}(c)$ of curvature $c=1,0$ or -1 . We choose a local field of orthonormal frames $e_{1}, \ldots, e_{m}$ in $R^{m}(c)$ such that, restricted to $M$, the vectors $e_{1}, e_{2}$ are tangent to $M$ (and, consequently, $e_{3}, \ldots, e_{m}$ are normal to $M$ ). With respect to the frame field of $R^{m}(c)$ chosen above, let $\omega^{1}, \ldots, \omega^{m}$ be the field of dual frames. Then the structure equations of $R^{m}(c)$ are given by

$$
\begin{aligned}
& d \omega^{A}=\sum \omega_{A}^{B} \wedge \omega^{B}, \quad \omega_{B}^{A}+\omega_{A}^{B}=0, \\
& d \omega_{B}^{A}=\sum \omega_{C}^{A} \wedge \omega_{C}^{B}+c \omega^{A} \wedge \omega^{B}, \quad A, B, C=1, \ldots, m .
\end{aligned}
$$

We restrict these forms to $M$. Then $\omega^{r}=0, r, s, t=3, \ldots, m$. Since

$$
0=d \omega^{r}=\omega_{r}^{1} \wedge \omega^{1}+\omega_{r}^{2} \wedge \omega^{2}
$$

by Cartan's lemma we may write

$$
\omega_{i}^{r}=\sum h_{i j}^{r} \omega j, \quad h_{i j}^{r}=h_{j i}^{r}, \quad i, j=1,2 .
$$

From these we obtain

$$
d \omega^{i}=\sum \omega_{i}^{j} \wedge \omega^{j}
$$




$$
\begin{aligned}
d \omega_{2}^{1} & =\left\{c+\sum_{r} \operatorname{det}\left(h_{i j}^{r}\right)\right\} \omega^{1} \wedge \omega^{2}=K \omega^{1} \wedge \omega^{2} . \\
d \omega_{i}^{r} & =\sum \omega_{j}^{r} \wedge \omega_{j}^{i}+\sum \omega_{s}^{r} \wedge \omega_{s}^{i} .
\end{aligned}
$$

The second fundamental form $\boldsymbol{h}$ and the mean curvature vector $\boldsymbol{H}$ are given respectively by

$$
\begin{aligned}
\boldsymbol{h} & =\sum h_{i j}^{r} \omega^{i} \wedge \omega^{j} \boldsymbol{e}_{r}, \\
\boldsymbol{H} & =\frac{1}{2} \sum h_{i i}^{r} \boldsymbol{e}_{r} .
\end{aligned}
$$

\section{Proof of Theorem 1}

Let $\alpha$ denote the mean curvature of $M$. We now consider the cases $\alpha>0$ and $\alpha=0$ separately.

Case (i) $\alpha>0$. In this case, we may choose our frame field in such a way that

$$
\begin{gathered}
H=\alpha e_{3}, \\
h_{12}^{r}=0, \text { for } r=3, \ldots, m .
\end{gathered}
$$

Since $M$ is pseudo-umbilical, we have

$$
\begin{gathered}
\omega_{i}^{3}=\alpha \omega^{i}, \\
\omega_{1}^{r}=h_{11}^{r} \omega^{1}, \quad \omega_{2}^{r}=-h_{11}^{r} \omega^{2}, \quad r=4, \ldots, m .
\end{gathered}
$$

By taking exterior differentiations of (13) and applying (6), (8) and (14), we obtain

$$
\sum_{r=4}^{m} h_{i i}^{r} \omega_{r}^{3} \wedge \omega^{i}=0, \text { for } i=1,2 .
$$

On the other hand, by taking exterior differentiations of (14) and applying (6), (8) and (13), we obtain

$$
d h_{i i}^{r} \wedge \omega^{i}+2 h_{i i}^{r} d \omega^{i}+\alpha \omega_{3}^{r} \wedge \omega^{i}=\sum_{s=4}^{m} h_{i i}^{s} \omega_{r}^{s} \wedge \omega^{i}
$$

for $r=4, \ldots, m$ and $i=1,2$. Multiplying (16) by $h_{i i}^{r}$ and summing up on $r$ from 4 to $m$, we obtain

$$
\begin{aligned}
\sum_{r=4}^{m} h_{i i}^{r} d h_{i i}^{r} \wedge d \omega^{i}+2 \sum_{r=4}^{m}\left(h_{i i}^{r}\right)^{2} d \omega^{i}+\alpha \sum_{r=4}^{m} h_{i i}^{r} \omega_{3}^{r} \wedge \omega^{i} & \\
& =\sum_{r, s=4}^{m} h_{i i}^{r} h_{i i}^{s} \omega_{r}^{s} \wedge \omega^{i}, \quad i=1,2 .
\end{aligned}
$$

By using $\omega_{s}^{r}+\omega_{r}^{s}=0$ and (15), we obtain

$$
\sum_{r=4}^{m} h_{i i}^{r} d h_{i i}^{r} \wedge d \omega^{i}+2 \sum_{r=4}^{m}\left(h_{i i}^{r}\right)^{2} d \omega^{i}=0, \quad i=1,2 .
$$

On the other hand, since the Gauss curvature $K$ is constant, we have

$$
\sum_{r=4}^{m}\left(h_{i i}^{r}\right)^{2}=c+\alpha^{2}+K=\text { constant. }
$$


Therefore, by (18) and (19) we obtain

$$
\left(c+\alpha^{2}+K\right) d \omega^{i}=0, \quad i=1,2 .
$$

If $c+\alpha^{2}+K=0$, then $h_{i i}^{r}=0$ for all $r>3$. This implies that $M$ is totally umbilical in $R^{m}(c)$. If $c+\alpha^{2}+K \neq 0$, then we obtain $d \omega^{1}=d \omega^{2}=0$ identically on $M$. Therefore, by (6), we obtain $\omega_{2}^{1}=0$ identically. This implies that $M$ is flat.

Case (ii) $\alpha=0$. In this case, by the fact that $K_{N}=0$, we may choose our frame field in such a way that

Hence, we have

$$
h_{12}^{r}=0, \text { for } r=3, \ldots, m \text {. }
$$

$$
\omega_{1}^{r}=h_{11}^{r} \omega^{1}, \quad \omega_{2}^{r}=-h_{11}^{r} \omega^{2}, \quad r=3, \ldots, m .
$$

Taking exterior differentiations of (22), we have

$$
d h_{11}^{r} \wedge \omega^{i}+2 h_{11}^{r} d \omega^{i}=\sum_{s=3}^{m} h_{11}^{s} \omega^{i} \wedge \omega_{s}^{r},
$$

for $r=3, \ldots, m$ and $i=1,2$. Multiplying (23) by $h_{11}^{r}$ and summing up on $r$, we obtain

$$
\sum_{r=3}^{m}\left(h_{11}^{r} d h_{11}^{r}\right) \wedge \omega^{i}+2 \sum_{r=3}^{m}\left(h_{11}^{r}\right)^{2} d \omega^{i}=0, \quad i=1,2 .
$$

On the other hand, the constancy of the Gauss curvature implies that the first term of (24) vanishes. Thus we obtain

$$
\sum_{r=3}^{m}\left(h_{11}^{r}\right)^{2} d \omega^{i}=0, \quad i=1,2 .
$$

This implies that $M$ is either totally geodesic or flat. Consequently, we see that, in both cases, $M$ is either flat or totally umbilical in $R^{m}(c)$. This proves the first part of the theorem. The second part follows immediately from the first part and the last paragraph of $\S 1$ of (2).

\section{Proof of Theorem 2}

Let $M$ be a compact surface with Gauss curvature $K \leqq 0$ in a space form $R^{m}(c)$. If there exists a non-degenerate normal vector field $e$ over $M$, which is perpendicular to the mean curvature vector $\boldsymbol{H}$, then we may choose our frame field in such a way that $e_{3}=e$ and $e_{1}, e_{2}$ are in the principal direction of $e$. Since $\boldsymbol{e}$ is perpendicular to the mean curvature vector field $\boldsymbol{H}$, we have

$$
\omega_{1}^{3}=g \omega^{1}, \quad \omega_{2}^{3}=-g \omega^{2}, \quad g>0 .
$$

The parallelism of $e$ in the normal bundle implies

$$
\omega_{r}^{3}=0, \text { for } r=4, \ldots, m \text {. }
$$

By taking exterior differentiations of (26) and applying (27) we obtain

$$
2 g d \omega^{i}+d g \wedge \omega^{i}=0, \quad i=1,2 .
$$


From (28) we can consider local coordinates $(u, v)$ in an open neighbourhood $U$ of a point $p \in M$ such that

$$
d s^{2}=E d u^{2}+G d v^{2}, \quad \omega^{1}=\sqrt{E} d u, \quad \omega^{2}=\sqrt{\bar{G}} d v,
$$

where $d s^{2}$ is the first fundamental form and $E$ and $G$ are local positive functions on $U$. From (28), equation (29) becomes

$$
d(g E) \wedge d u=0, \quad d(g G) \wedge d v=0,
$$

which shows that $(g E)$ is a function of $u$ and $(g G)$ is a function of $v$. By making the following coordinate transformation:

$$
u^{\prime}=\int(g E)^{\frac{1}{t}} d u, \quad v^{\prime}=\int(g G)^{\frac{1}{2}} d v,
$$

we see that there exists a neighbourhood $V$ of each point $p \in M$ such that there exist isothermal coordinates $(u, v)$ in $V$ such that

$$
\left\{\begin{array}{l}
d s^{2}=f\left\{d u^{2}+d v^{2}\right\}, \quad \omega^{1}=\sqrt{f} d u, \quad \omega^{2}=\sqrt{f} d v, \\
g f=1
\end{array}\right.
$$

where $f=f(u, v)$ is a positive function defined on $V$. It is well-known that the Gauss curvature $K$ is given by

$$
K=-\frac{1}{2 f} \Delta \log (f),
$$

with respect to the isothermal coordinates $(u, v)$. Hence the condition $K \leqq 0$ with $g f=1$ implies $\Delta \log (g)=-\Delta \log (f) \leqq 0$. By Hopf's lemma, we see that $\log (g)$ is a constant on $M$. Hence the Gauss curvature

$$
K=-\frac{1}{2 f} \Delta \log (f)=\frac{g}{2} \Delta \log (g)=0 .
$$

This implies that $M$ is flat. By taking exterior differentiation of (29) we obtain

$$
\omega_{1}^{3} \wedge \omega_{1}^{r}+\omega_{2}^{3} \wedge \omega_{2}^{r}=0, \text { for } r>3 .
$$

Substituting (26) into (34) we obtain $g h_{12}^{r}=0$, for $r>3$. This implies $K_{N}=0$. This completes the proof of the theorem.

\section{REFERENCES}

(1) B.-Y. Chen, Minimal surfaces of $S^{m}$ with Gauss curvature $\leqq 0$, Proc. Amer. Math. Soc. 31 (1972), 235-238.

(2) B.-Y. CheN, Pseudo-umbilical submanifolds of a Riemannian manifold of constant curvature, III, J. Differential Geometry (to appear).

(3) B.-Y. Chen and G. D. LudDen, Rigidity theorems for surfaces in euclidean space, Bull. Amer. Math. Soc. 78 (1972), $72-73$. 
(4) S. S. Chern, M. do Carmo and S. Kobayashi, Minimal submanifolds of a sphere with second fundamental form of constant length, Functional Analysis and Related Fields (Springer-Verlag, 1970), 60-75.

(5) H. B. Lawson, Local rigidity theorems for minimal hypersurfaces, Ann. of Math. (2) 89 (1969), 187-197.

Michigan State University

East Lansing, Michigan 48823 